\title{
Long-term memory for abstract concepts in the lowland gorilla (Gorilla g. Gorilla)
}

\author{
THOMAS L. PATTERSON and OVID J. L. TZENG \\ University of California, Riverside, California 92521
}

\begin{abstract}
Four gorillas underwent a series of experiments on discrimination reversal (DR) problems. Upon achieving learning criterion, they were tested with various types of transfer conditions. The results suggested that during DR training the gorillas learned an abstract strategy. This acquired abstract concept was retained for at least 2.5 years. A further experiment showed that the gorillas' long-term memory was not due to the specific stimuli used.
\end{abstract}

The study of memory in nonhuman primates is not only interesting in its own right, but also, through the use of these subjects we may gain a better understanding of human memory. Because of their evolutionary similarities to humans, primates have received increasing attention in recent behavioral and physiological research. Since this group of animals often provides the basis for generalization about man, it is important to develop an adequate understanding of their behavioral capacities. As Rumbaugh (1970) pointed out, "performance of nonverbal animals is now of great interest and it is intriguing to define the maximum delays through which they can in some manner retain information vital to eventual performance of the correct choice" (p. 30). However, animal studies have in the past rarely been directly at the problem of memory, which has always been conceived as a "higher order mental process." Even in the 1970s, there are animal psychologists, haunted by Aristotle's assertion that animals are incapable of higher mental processes and by the "mental block" arising from their behavioristic tradition, who are still puzzling over the question, "Do animals have memory?" (Ruggiero \& Flagg, 1976; Winograd, 1971).

In the past several years, the advancement in human memory research, both in terms of experimental findings and of theoretical formulations, has attracted many animal psychologists. They began to apply the experimental paradigms and memory models developed in human memory research to the study of animal behavior. Terms such as encoding, storage, and retrieval have also been transferred directly from human memory models to the descriptions of the "processes" involved in animal memory. A good example of this is the recent interest in "short-term" memory in animals (Ruggiero \& Flagg, 1976). While the results of many such applications are both fruitful and interesting, they nevertheless

We thank D. M. Rumbaugh for sending us the original acquisition data. His support during various phases of this study is acknowledged with deep appreciation. Reprint requests should be sent to either Thomas L. Patterson or Ovid Tzeng of the Department of Psychology, University of California, Riverside, California 92521. reveal a major problem: The term "short-term memory" may be a misnomer in animal studies. In fact, in most cases the term is used to reflect a certain type of experimental paradigm patterned after that of human memory research rather than a theoretical construct. This differs from its usage in human memory research, where shortterm memory describes an intermediate stage between the encoding of stimuli and the establishment of a more endurable trace or "long-term memory." The viability of postulating such an intermediate stage depends on the validity of a long-term memory and on the differential functional properties associated with the postulated short- and long-term memories. In human memory research, the validity of a long-term memory can be established by phenomenological evidence in our everyday lives as well as by empirical evidence that we do indeed remember things or events over many decades (Bahrick, Bahrick, \& Wittlinger, 1975; Squire \& Slater, 1975). It is difficult to draw a parallel case in animal research to substantiate the claim for such a long-term memory. Thus, until the concept of a long-term memory can be empirically verified in animals, the term shortterm memory will remain an indication of a particular type of experimental procedure rather than a useful theoretical construct.

The purpose of the present study is to provide some direct and clear evidence of long-term (2.5 years) memory in four gorilla subjects. The earliest report of testing gorilla memory in a rigorous experimental setting was given by Yerkes (1928) on a single gorilla subject in the Ringling Brothers and Barnum and Bailey Circus. He reported that his subject was able to respond correctly in a delayed response experiment after delays of at least $10 \mathrm{~min}$, and in a buried food experiment to remember the food's presence and locate it after intervals of 1 to 2 days. Though interesting, Yerkes' results were hardly satisfactory for at least two reasons. First, the delayed response procedure has been shown to be problematic (Fletcher, 1965). Yerkes himself noted that the major problem with this procedure is that it is hard to know that the subject is indeed paying attention to the stimulus presentation. Second, the task was too simple to 
allow any argument for memory of a complex nature. Furthermore, the result of retention over $10 \mathrm{~min}$ is by no means a convincing demonstration of long-term memory. Yerkes also noticed this difficulty and urged further studies to determine accurately the temporal span of the gorilla's memory.

Following the pioneer work of Yerkes (1928), Rumbaugh and his associates (Rumbaugh, 1971; Rumbaugh \& Steinmetz, 1971) used a modified discrimination-reversal (DR) task, which he believed circumvented most of the shortcomings associated with the delayed response paradigm, to determine whether qualitative differences exist in the learning processes of gorillas, gibbons, and talapoins at the San Diego Zoo. Rumbaugh's results showed that among the three kinds of animals, gorillas are particularly capable of abstract forms of learning. The present study is a followup of Rumbaugh's study. Two and one-half years after the original testing, we retested the four gorilla subjects on similar discrimination problems. The testing situation, experimenter, and all other conditions are the same for the present study as they were in a previous study reported by Rumbaugh (1971). The subjects underwent no testing in the interim between data collection periods. A series of experiments were conducted to test the lowland gorilla's ability to retain an abstract concept over a 2.5-year period.

\section{METHOD}

\section{Subjects}

The subjects were four juvenile gorillas (Gorilla g. gorilla) from the San Diego Zoo's private collection. The group was made up of two males (Mimbo and Junior) and two females (Dolly and Timbo)

\section{Procedure}

The entire study can be divided into three separate stages. The first was a DR learning stage in which the subjects received a common set of 100 two-choice discrimination problems in a modified Wisconsin General test apparatus (Harlow, 1949). In a DR task, responses to one stimulus are consistently rewarded and responses to the other stimulus are consistently not rewarded. After a predetermined number of trials, the cue values of the stimuli are reversed, that is, only choice of the initially incorrect stimulus is rewarded. The purpose of this stage was to demonstrate that, indeed, the gorilla subjects are capable of such DR learning.

The second stage immediately followed the first and was concerned mainly with the question of what was learned during the DR training. The question focused on whether the gorillas were engaging in abstract learning that cannot be accounted for by a S-R theory. Three types of transfer condition were given to each subject on the following day. On each DR problem, subjects were trained to the criterion of nine correct responses within 10 consecutive trials. Upon subjects' achievement of the criterion, the cue values of the stimuli were reversed for the first of the 11 reversal trials given per problem. In other words, with $\mathrm{A}+$ representing the correct stimulus and $\mathrm{B}-$ the incorrect stimulus for each problem used in criterion training, $A+$ became $\mathrm{A}-$ and $\mathrm{B}$ - became $\mathrm{B}+$ for the first reversal trial. This first trial served as the reversal cue for these DR-sophisticated subjects. On the following 10 reversal trials of each problem, the substitution of a new stimulus, $C$, for either the initially correct Stimulus A or the initially incorrect Stimulus B, in two of three transfer conditions, was intended to reveal the nature of the DR learning during acquisition. In the third condition, which serves as control, both stimuli, initial A+ and B-, were retained for all 10 reversal trials as $\mathrm{A}$ - and $\mathrm{B}+$. The purpose of the second stage was to demonstrate that the gorilla subjects were engaging in an abstract learning rather than simple S-R associations. Detailed analyses of the first and second stages are presented in Rumbaugh and Steinmetz (1971).

The third stage was a retention test. A total of 2.5 years after the completion of the second stage, all four subjects were once again given the exact same three types of transfer problems as in the second stage. The purpose was to see whether they still remembered what they had learned after a retention interval of 2.5 years.

\section{RESULTS AND DISCUSSION}

The results of the experiment at the first stage showed that subjects' DR performance improved from an initial level of about 32\% correct on Problems 1-20 to about $75 \%$ correct on Problems 21-100. Clearly, gorillas are capable of DR learning.

The results of transfer learning at the second stage (solid lines) are plotted in Figure 1 together with the results obtained at the third stage (broken lines). The data on each type of transfer conditions are shown separately. Note that if the prereversal criterion had been achieved, reversal required concurrent extinction of $A$ and habit strength accrual to $B$, then discarding of A with substitution of a new stimulus, $\mathrm{C}-$, should expedite the reversal process; extinction of the habit to A would be obviated. Similarly, discarding of B and substitution of it with $\mathrm{C}+$ dispenses with the inhibition that should accrue to $\mathrm{B}$ - during criterional training in proportion to the number of trials it was chosen. Thus, according to simple S-R theory, both conditions involving a new stimulus, $\mathrm{C}$, should allow for better reversal performance than the control condition. However, if through the course of prereversal criterional training and the reversal trials, a relatively abstract learning

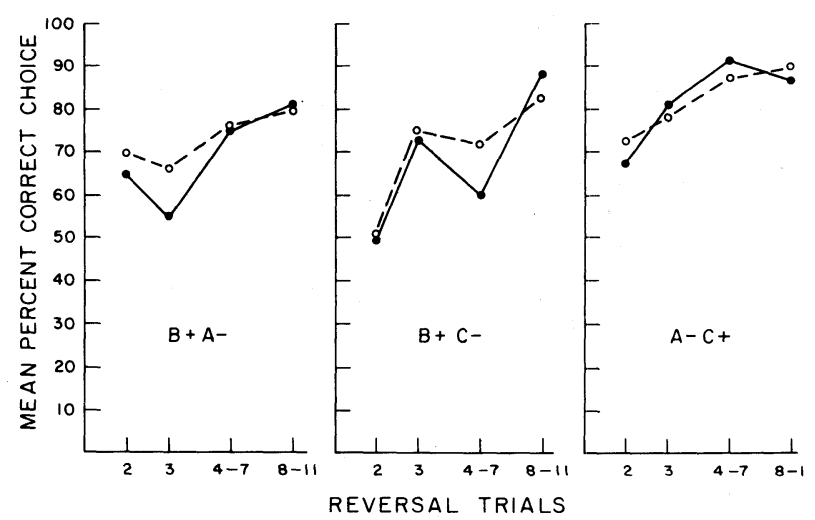

Figure 1. Mean percent correct choices on the three types of reversal problems as a function of the number of reversal trials for the immediate test (solid line) and the $\mathbf{2 . 5}$ year delayed test (broken line). 
developed that involved a wir-stay, lose-shift strategy (Levine, 1959) that would take into account the contingencies of all reversal conditions, then performance on all three transfer conditions should be easy and relatively equal. As the three solid lines in Figure 2 show, the results of this transfer experiment showed that the three reversal conditions were essentially equivalent and relatively easy, suggesting that the gorillas were engaging in abstract learning. That is, it seems that these gorillas adopted a win-stay, lose-shift strategy as discussed by Levine (1959) to solve the DR problems: Given a loss on Trial 1 of reversal with a choice of A, which was previously + , and then on subsequent trials (1) given re-presentation of $A$, shift from $A$ to any alternative afforded (B or C) or (2) given no representation of $A$, shift to $B$ disregarding any alternative (C) (Rumbaugh, 1971).

In his original study, Rumbaugh (1971) compared gorillas' transfer learning results with those of gibbons (Hylobates lar) and of talapoins (Miopithecus talapoin). $\mathrm{He}$ found that under the three transfer conditions described above, the latter two groups produced totally different transfer patterns, suggesting that both gibbons and talapoins are more S-R associational learners. From these comparative data, Rumbaugh concluded that of the nonhuman primates gorillas are certainly capable of abstract forms of learning. One immediate question of interest is whether or not such abstract learning would be retained over a long period. This question can be answered by examining the broken lines in Figure 1, which represent a 2.5 year retention after the original learning. Clearly, the gorillas performed as well as they did before. More importantly, the three types of transfer problems were equally easy for them, suggesting once again that what they remembered was indeed some abstract concept.

One objection to the above conclusion can be made: Since the same transfer problems were employed at both the second and third stages, subjects might just remember the specific stimuli used. In order to test the validity of this objection, another experiment was conducted. A totally new set of stimuli was constructed. All four subjects were then given a new series of reversal problems. In half of the problems the unfamiliar stimuli were used, and in the remaining half familiar stimuli were used. The procedure was identical to that of the second and third stages except that only the standard reversal problems (i.e., $\mathrm{A}+\mathrm{B}-$ shift to $\mathrm{A}-\mathrm{B}+$ ) were tested. The results of this experiment are presented in Figure 2. Figure 2a shows that there is no difference in prereversal acquisition performance between DR problems with the familiar stimuli and those with the unfamiliar stimuli. This pattern of "null" difference is further substantiated by Figure $2 b$, which shows that performance on the reversal trials was nearly equal between those trials using the familiar stimuli and those using the unfamiliar stimuli. Evidently, the gorillas
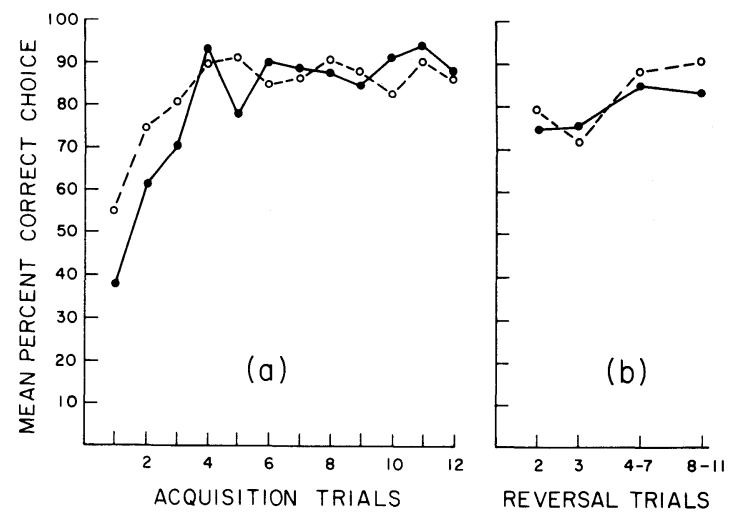

Figure 2. Mean percent correct choices for problems using familiar stimuli (solid line) and problems using unfamiliar stimuli (broken line) as a function of (a) the number of prereversal acquisition trials and (b) the number of postreversal trials.

remembered the abstract concept rather than the specific stimuli used 2.5 years before.

In summary, the present study not only shows that gorillas are capable of abstract learning, but also that they remembered this abstract concept over a very long period of time. Empirically, this retention interval, 2.5 years, is the longest yet reported in the literature for any nonhuman primate. Theoretically, the demonstration of a long-term "semantic" memory in animals other than humans enlightens the possibility of applying human memory models to animal research. Like the promising results reported in teaching the American Sign Language to some nonhuman primates (Gardner \& Gardner, 1969; Patterson, 1978), the results of the present study suggest that the gap between human and nonhuman primates is not the chasm it was once believed to be.

\section{REFERENCES}

Bahrick, H. P., Bahrick, P. O., \& Wittllinger, R. P. Fifty years of memory for names and faces: A cross-sectional approach. Journal of Experimental Psychology: General, 1975, 1, 54-75.

Fletcher, H. J. The delayed-response problem. In A. Schrier, H. Harlow, \& F. Stollnitz (Eds.), Behavior of non-human primates (Vol. 1). New York: Academic Press, 1965.

GARDNER, R. A., \& GARDNER, B. T. Teaching sign language to a chimpanzee. Science, 1969, 165, 664-672.

Harlow, H. F. The formation of learning sets. Psychological Review, 1949, 56, 51-56.

LEVINE, M. A model of hypothesis behavior in discrimination learning set. Psychological Revew, 1959, 66, 353-366.

Patterson, F. G. The gestures of a gorilla: Language acquisition in another pongid. Brain and Language, 1978, 5, 72-97.

Ruggiero, F. T., \& FlaGG, S. F. Do animals have memory? In D. L. Medin, W. A. Roberts, \& R. T. Davis (Eds.), Processes of animal memory. Hillsdale, N.J: Erlbaum, 1976.

Rumbaugh, D. M. Learning skills of anthropoids. In L. A. Rosenblum (Ed.), Primate behavior: Development in field and laboratory research. New York: Academic Press, 1970. 
Rumbaugh, D. M. Evidence of qualitative differences in learning processes among primates. Journal of Comparative and Physiological Psychology, 1971, 76, 250-255.

Rumbaugh, D. M., \& Steinmetz, G. T. Discrimination reversal skills of the lowland gorilla (Gorilla g. gorilla). Folia Primatologica, 1971, 16, 144-152.

SQUIRE, L. R., \& Slater, P. C. Forgetting in very long-term memory as assessed by an improved questionnaire technique. Journal of Experimental Psychology: Human Learning and Memory, 1975, 1, 50-54.
WINOGRAD, E. Some issues relating animal memory to human memory. In K. H. Honig \& P. H. R. James (Eds.), Animal memory. New York: Academic Press, 1971.

Yerkes, R. M. The mind of a gorilla. Comparative Psychology Monographs, 1928, 5, 1-91.

(Received for publication January 31, 1979.) 